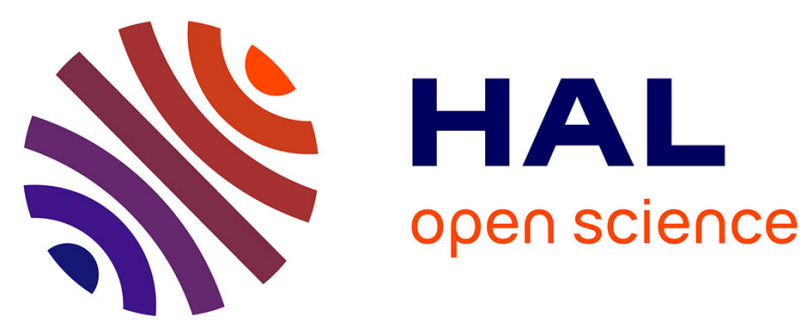

\title{
CFAR property and robustness of the lowrank adaptive normalized matched filters detectors in low rank compound gaussian context
}

\author{
Arnaud Breloy, Guillaume Ginolhac, Frédéric Pascal, Philippe Forster
}

\section{To cite this version:}

Arnaud Breloy, Guillaume Ginolhac, Frédéric Pascal, Philippe Forster. CFAR property and robustness of the lowrank adaptive normalized matched filters detectors in low rank compound gaussian context. 2014 IEEE 8th Sensor Array and Multichannel Signal Processing Workshop (SAM 2014), Jun 2014, La Corogne, Spain. pp.301 - 304, 10.1109/SAM.2014.6882401 . hal-01059726

\section{HAL Id: hal-01059726 \\ https://hal.science/hal-01059726}

Submitted on 1 Sep 2014

HAL is a multi-disciplinary open access archive for the deposit and dissemination of scientific research documents, whether they are published or not. The documents may come from teaching and research institutions in France or abroad, or from public or private research centers.
L'archive ouverte pluridisciplinaire HAL, est destinée au dépôt et à la diffusion de documents scientifiques de niveau recherche, publiés ou non, émanant des établissements d'enseignement et de recherche français ou étrangers, des laboratoires publics ou privés. 


\title{
CFAR PROPERTY AND ROBUSTNESS OF THE LOW RANK ADAPTIVE NORMALIZED MATCHED FILTERS DETECTORS IN LOW RANK COMPOUND GAUSSIAN CONTEXT
}

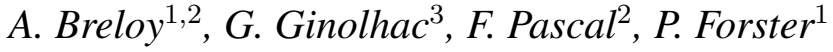 \\ 1 SATIE, ENS Cachan, CNRS, UniverSud, 61, av President Wilson, F-94230 Cachan, France \\ 2 SONDRA, SUPELEC, Plateau du Moulon, 3 rue Joliot-Curie, F-91192 Gif-sur-YvetteCedex, France \\ ${ }^{3}$ LISTIC, Université de Savoie, BP 80439, 74944 ANNECY LE VIEUX Cedex, France
}

\begin{abstract}
In the context of an heterogeneous disturbance with a Low Rank (LR) structure (referred to as clutter), one may use the LR approximation for detection process. Indeed, in such context, adaptive LR schemes have been shown to require less secondary data to reach equivalent performances as classical ones. The LR approximation consists on cancelling the clutter rather than whitening the whole noise. The main problem is then the estimation of the clutter subspace instead of the noise covariance matrix itself. Maximum Likelihood estimators (MLE), under different hypothesis [1][2][3], of the clutter subspace have been recently proposed for a noise composed of a LR Compound Gaussian (CG) clutter plus a white Gaussian Noise (WGN). This paper focuses on the performances of the LR Adaptive Normalized Matched Filter (LR-ANMF) detector based on these different clutter subspace estimators. Numerical simulations illustrate its CFAR property and robustness to outliers.
\end{abstract}

Index Terms - Covariance Matrix Estimation, Maximum Likelihood, Low Rank, ANMF Detector, Compound Gaussian, STAP.

\section{INTRODUCTION}

In most radar applications the additive disturbance can be modeled by a sum of two noises: a White Gaussian Noise (WGN), due to electronics, and the so-called clutter, the response of the environment to the emitted signal. In some contexts, the clutter is known to have la Low rank (LR) structire, i.e. to have a singular Covariance Matric (CM) of rank $R \ll M$ (with $M$ the dimension of the data). In practice, this LR structure of the disturbance can be exploited to build LR processes. The approach consists in canceling the clutter instead of performing a classical whitening the noise. Thus LR methods are not based on the estimated noise $\mathrm{CM}$ but on the estimated clutter subspace projector (CSP) only, usually derived from an SVD of a CM estimate. LR adaptive techniques present two main advantages. Firstly, estimating the CSP requires less secondary data to ensure good performance. For example, only $K \geq 2 R$ secondary data are needed to ensure a classical $3 \mathrm{~dB}$ loss of the output SNR compared to optimal fil- tering [4], while classical filter requires $K \geq 2 M$ secondary data to reach equivalent performance [5]. Secondly, LR methods are robust to secondary data contamination by outliers [6].

Classically the LR clutter has been modeled by a correlated Gaussian noise with eigenvalues that largely exceeds the power of the WGN, leading to the Sample Covariance Matrix (SCM) as estimator of the CM and CSP. Nevertheless, the SCM is not well adapted in highly heterogeneous or impulsive clutter environment. Therefore, developing filters/detectors based on it may lead to poor performance. To describe an heterogeneous clutter, one of the most general model is provided by the Complex Elliptically Symmetric distribution (CES) [7]. Among the general CES class, this paper will focus on the Compound-Gaussian (CG) distributions $^{1}$ that covers a large panel of well known heavy-tailed distributions. Eventually, the disturbance will be modeled in this paper as a LR-CG clutter plus WGN.

In that context, one can derive the CSP from the SVD of a robust estimate of the $\mathrm{CM}$, such as M-estimators [7] or the Fixed Point Estimator (FPE)[8]. However, these estimators require $K>M$ to be computed, which does does not allow to take fully advantage of the LR hypothesis if $2 R \ll M$. A currently an active topic of research focuses on regularization of the algorithms to compute these estimators in under sampled configurations [9][10]. Nevertheless, with regard to the considered model of LR-CG plus WGN, recent results are providing direct CSP estimators [1][2][3]. These estimators are derived from a intermediate matrix that is not necessarily an estimate of the CM and can be computed when $K>R$.

For the considered disturbance, the LR Normalized Matched Filter (LR-NMF) detector and its theoretical performance have been introduced in [11]. Its adaptive version, the LR-ANMF, have also been investigated [11][6]. However, the choice of the appropriate CSP estimator to perform LR adaptive detection remains an opened question. In this paper, we propose then to study the performance and robustness of the LR-ANMF build from different CSP estimators: derived from a classical approach (SCM, NSCM), derived

\footnotetext{
${ }^{1}$ Also referred to as Spherically Invariant Random Vectors (SIRV) in the literature
} 
from an MLE approach [2][3] and derived from a regularized robust estimator [10]. The studied radar configuration is an airborne Space Time Adaptive Processing (STAP) [12], since the Brennan Rule [13] shows that the clutter rank is satisfying $R \ll M$ in this application. Results are derived from Monte Carlo simulations and also an illustration from a real data set.

\section{SIGNAL MODEL AND LR DETECTOR}

The stated problem is to infer if the received signal $\mathbf{z}$, corrupted by an additive disturbance $\mathbf{n}$, also contains a complex known signal $\mathbf{d}$. One also have a set of $K$ secondary data $\{\mathbf{z}\}_{k}$ which are signal free realizations of the disturbance. The two hypothesis are then:

$$
\left\{\begin{array}{lll}
H_{0}: \mathbf{z}=\mathbf{n}, & \mathbf{z}_{k}=\mathbf{n}_{k} k, \in \llbracket 1, K \rrbracket \\
H_{1}: \mathbf{z}=\mathbf{d}+\mathbf{n}, & \mathbf{z}_{k}=\mathbf{n}_{k}, k \in \llbracket 1, K \rrbracket
\end{array}\right.
$$

The additive disturbance is the sum of the ground clutter $\mathbf{c}$ and a thermal noise $\mathrm{g}$ :

$$
\mathbf{n}=\mathbf{c}+\mathbf{g}
$$

The thermal noise is modeled by a WGN of known power $\sigma^{2}$ i.e. $\mathbf{n} \sim \mathcal{C} \mathcal{N}\left(\mathbf{0}, \sigma^{2} \mathbf{I}_{m}\right)$. The hypothesis of known $\sigma^{2}$ is made for describing a valid theoretical framework. In practice, presented results could be applied with a prior estimate of $\sigma^{2}$ used as its actual value. The ground clutter is an heterogeneous noise that has a different power in each cell $k$. The randomness of its power is induced by spatial variation in the radar backscattering. In such a situation, it is common to model this kind of clutter by a CG process [7]. A realization of a CG process corresponds to a Gaussian random vector multiplied the square root of a random power factor called the texture $\tau$ of Probability Density Function (PDF) $f_{\tau}$. Moreover, in side looking STAP, the rank $R$ of the clutter $\mathrm{CM}$ can be evaluated [13] and is verifying $R \ll M$. One has then $\mathbf{c} \sim \mathcal{C G}\left(\mathbf{0}, \boldsymbol{\Sigma}_{c}, f_{\tau}\right)$. With the rank $R$ clutter $\mathrm{CM}$ defined by its eigendecomposition:

$$
\boldsymbol{\Sigma}_{c}=\sum_{r=1}^{R} c_{r} \mathbf{v}_{r} \mathbf{v}_{r}^{H}
$$

The whole noise covariance matrix is then defined as

$$
\boldsymbol{\Sigma}=\sigma^{2} \mathbf{I}_{M}+E(\tau) \boldsymbol{\Sigma}_{c}
$$

However, in a realistic STAP application, no prior information is available on the $\operatorname{PDF} f_{\tau}$. In that case, each secondary data may be described as $\mathbf{z}_{k} \sim \mathcal{C N}\left(\mathbf{0}, \boldsymbol{\Sigma}_{k}\right)$, with

$$
\boldsymbol{\Sigma}_{k}=\sigma^{2} \mathbf{I}_{M}+\tau_{k} \boldsymbol{\Sigma}_{c},
$$

where the textures of each realizations $\tau_{k}$ are considered as unknown deterministic parameters.
Usual detection processes require the noise CM $\Sigma$. However, considering the described framework, one can exploit the LR structure of the noise and cancel the clutter instead of whitening it. Adaptive LR processes are therefore based on the following LR approximation:

$$
\boldsymbol{\Sigma}^{-1} \sim \frac{1}{\sigma^{2}} \boldsymbol{\Pi}_{c}^{\perp} \propto \boldsymbol{\Pi}_{c}^{\perp}
$$

where $\Pi_{c}^{\perp}$ is the projector onto the clutter subspace complementary and $\boldsymbol{\Pi}_{c}$ is the rank $R$ CSP, constructed from the eigenvectors of the clutter CM:

$$
\boldsymbol{\Pi}_{c}^{\perp}=\mathbf{I}_{M}-\boldsymbol{\Pi}_{c}=\mathbf{I}_{M}-\sum_{r=1}^{R} \mathbf{v}_{r} \mathbf{v}_{r}^{H}
$$

Leading to the LR-Normalized Matched Filter as detection test:

$$
\Lambda_{L R-N M F}=\frac{\left(\mathbf{d}^{H} \boldsymbol{\Pi}_{c}^{\perp} \mathbf{z}\right)^{2}}{\left(\mathbf{d}^{H} \boldsymbol{\Pi} \perp_{c}^{\perp} \mathbf{d}\right)\left(\mathbf{z}^{H} \boldsymbol{\Pi} \perp \mathbf{z}\right)} \underset{H_{0}}{\stackrel{H_{1}}{\gtrless}} \delta_{L R-N M F}
$$

In real case, the CSP is unknown and has to be estimated with the secondary data $\{\mathbf{z}\}_{k}$ to process adaptive detection. The interest of the LR approximation is that it needs less secondary data to reach equivalent performances as classical schemes [4], typically $K \sim 2 R$ instead of $K \sim 2 M$. Classically, an estimator of the CSP $\hat{\boldsymbol{\Pi}}_{c}$ is derived from the SVD of an estimate of the noise CM $\hat{\Sigma}$. The LR-ANMF is then defined by:

$$
\hat{\Lambda}_{L R-A N M F}=\frac{\left(\mathbf{d}^{H} \hat{\boldsymbol{\Pi}}_{c}^{\perp} \mathbf{z}\right)^{2}}{\left(\mathbf{d}^{H} \hat{\boldsymbol{\Pi}} \perp \mathbf{d}\right)\left(\mathbf{z}^{H} \hat{\boldsymbol{\Pi}}_{c}^{\perp} \mathbf{z}\right)} \gtrless_{H_{0}}^{H_{1}} \delta_{L R-A N M F}
$$

\section{CLUTTER SUBSPACE ESTIMATORS}

This section simply recalls the expression of the estimators that are going to be tested. For a more detailed review of their related model, properties and computation methods, we refer the reader to the associated references.

definition 1 The classical Sample Covariance Matrix (SCM), which is the MLE of the CM in a Gaussian context is:

$$
\hat{\mathbf{\Sigma}}_{S C M}=\frac{1}{K} \sum_{k=1}^{K} \mathbf{z}_{k} \mathbf{z}_{k}^{H}
$$

The projector estimate derived from the SVD of the SCM will be denoted $\hat{\Pi}_{S C M}$.

definition 2 The Normalized SCM (NSCM) is defined by:

$$
\hat{\mathbf{\Sigma}}_{N S C M}=\frac{1}{K} \sum_{k=1}^{K} \frac{\mathbf{z}_{k} \mathbf{z}_{k}^{H}}{\mathbf{z}_{k}^{H} \mathbf{z}_{k}}
$$

The NSCM is biased estimate of the CM, however it has been shown that it provides a consistent and robust estimate of the CSP [14][6]. The projector estimate derived from the SVD of the NSCM will be denoted $\hat{\mathbf{\Pi}}_{N S C M}$. 
In this paper, since we consider the case $K=2 R<M$, the FPE cannot be defined. However, it can be computed it with a regularization algorithm:

definition 3 The Shrinkage-FPE (SFPE), also known as Diagonnaly-Loaded FPE [9][10], is defined for $\beta \in] 0,1]$ by the fixed point equation:

$$
\hat{\mathbf{\Sigma}}_{S-F P E}(\beta)=(1-\beta) \frac{M}{K} \sum_{k=1}^{K} \frac{\mathbf{z}_{k} \mathbf{z}_{k}^{H}}{\mathbf{z}_{k}^{H} \hat{\boldsymbol{\Sigma}}_{S-F P E}^{-1}(\beta) \mathbf{z}_{k}}+\beta \mathbf{I}_{M}
$$

The projector estimate derived from the SVD of the S-FPE will be denoted $\hat{\Pi}_{S-F P E}$.

definition 4 Under the assumption of equals eigenvalues of the clutter CM, the approached MLE of the CSP (A-MLE) [2], denoted $\hat{\boldsymbol{\Pi}}_{A-M L E}$, is the projector onto the subspace defined by the $R$ strongest eigenvectors of the matrix:

$$
\hat{\mathbf{R}}=\sum_{k=1}^{K} \frac{\hat{\tau}_{k}}{\sigma^{2}+\hat{\tau}_{k}} \mathbf{z}_{k} \mathbf{z}_{k}^{H}
$$

with the estimated textures:

$$
\hat{\tau}_{k}=\left\{\begin{aligned}
\left\|\hat{\boldsymbol{\Pi}}_{c} \mathbf{z}_{k}\right\|^{2} / R-\sigma^{2} & \text { if }\left\|\hat{\mathbf{\Pi}}_{c} \mathbf{z}_{k}\right\|^{2}>R \sigma^{2} \\
0 & \text { else }
\end{aligned}\right.
$$

This estimator's expression stands when there is no prior information on the texture PDF, which is more realistic for a STAP application. However, the case of known texture PDF is treated in [1].

definition 5 Under the assumption of high CNR, the MLE of the CSP is the projector onto the subspace defined by the $R$ strongest eigenvectors of the LR-FPE [3], defined for for $K>$ $R$ as:

$$
\hat{\boldsymbol{\Sigma}}_{L R-F P E}=\frac{M}{K} \sum_{k=1}^{K} \frac{\mathbf{z}_{k} \mathbf{z}_{k}^{H}}{\mathbf{z}_{k}^{H} \hat{\boldsymbol{\Sigma}}_{L R-F P E}^{\dagger R} \mathbf{z}_{k}},
$$

where $^{\dagger_{R}}$ is the rank $R$ pseudo inverse operator. The projector estimate derived from the SVD of the LR-FPE will be denoted $\hat{\Pi}_{L R-F P E}$.

For the rest of the paper, the adaptive detectors $\hat{\Lambda}_{L R}$ will be denoted with the same index as the CSP estimate they are build from. For example $\hat{\Lambda}_{S C M}$ denotes the LR-ANMF build from $\hat{\Pi}_{S C M}$.

\section{SIMULATION RESULTS}

This section presents Monte-Carlo simulations for a realistic STAP configuration. STAP is a technique used in airborne phased array radar to detect moving target embedded in an interference background such as jamming or strong clutter [12]. The radar receiver consists in an array of $Q$ antenna elements processing $P$ pulses in a coherent processing interval. It is
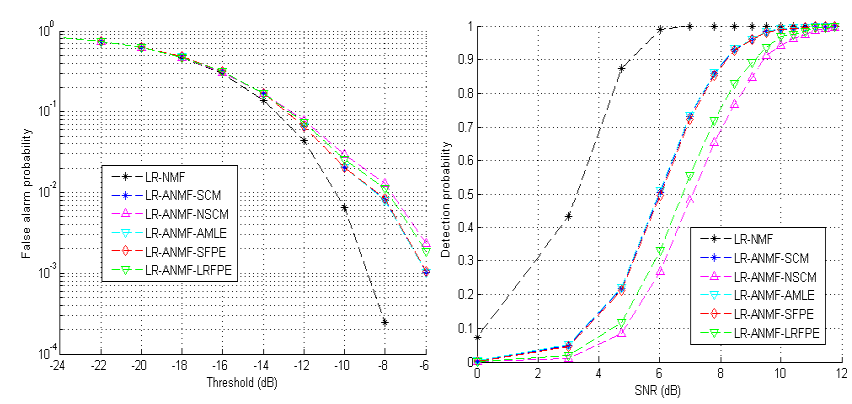

Fig. 1. PFA versus threshold (left) and the PD versus the $S N R$ for fixed PFA of $10^{-2}$ (right). STAP configuration: $Q=8, P=8, M=P Q=64$. Center frequency $f_{0}=450 \mathrm{MHz}$, bandwidth $B=4 \mathrm{MHz}$. Radar velocity $v=100 \mathrm{~m} / \mathrm{s}$. Inter-element spacing $d=\frac{c}{2 f_{0}}$ with $c$ the celerity of light. Pulse repetition frequency $f_{r}=600 \mathrm{~Hz} . R=15$ computed from Brennan rule [13].The texture PDF is a Gamma law of shape parameter $\nu=1$ and scale parameter $\frac{1}{\nu} . \mathrm{CNR}=30 \mathrm{~dB}$. Target speed $V_{T}=35 \mathrm{~m} / \mathrm{s}$ and angle $\phi_{T}=10^{\circ}$. The number of secondary data is set to $K=2 R$.
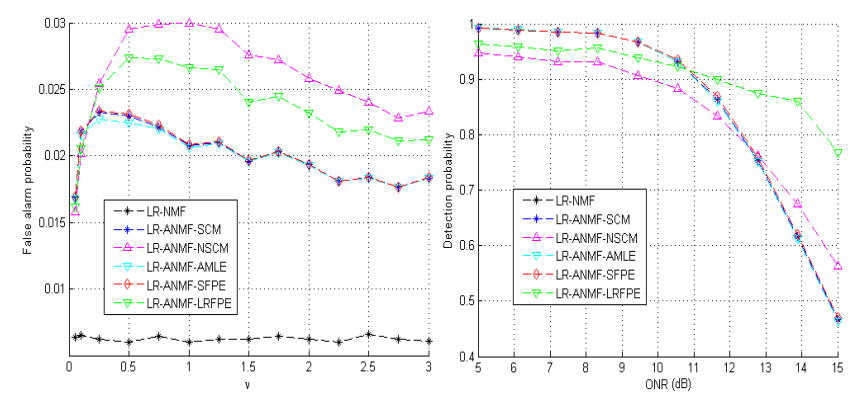

Fig. 2. CFAR test (left): PFA versus $\nu$ for a fixed threshold. Robustness test (right): PD versus ONR. Same STAP configuration.

important to notice that application fits the considered model since in side looking STAP, the clutter CM is known to be LR. Moreover, the rank can be evaluated thanks to the Brennan Rule [13].

Figure 1 shows both the Probability of False Alarm (PFA) versus the threshold of the detector and the Pobability of Detection (PD) versus the $S N R$ for a fixed PFA of $10^{-2}$. These figures illustrate that for a fixed PFA, the best PD is achieved indifferently with $\hat{\Lambda}_{S C M}, \hat{\Lambda}_{S-F P E}$ or $\hat{\Lambda}_{A-M L E}$. This shows that the hypothesis of equals eigenvalues of the CM does not strongly impacts the performance of $\hat{\Lambda}_{A-M L E}$. The performance of $\hat{\Lambda}_{L R-F P E}$ is below, meaning that the high CNR hypothesis is probably not satisfied enough, but is still better than $\hat{\Lambda}_{N S C M}$. Figure 2 right shows Probability of False Alarm (PFA) versus $\nu$ for a fixed threshold: it presents an illustration of the relatively (acceptable for $\nu>0.25$ ) CFAR property of the LR-ANMF. In Figure 2 left, the steering vector of the target is inserted into the secondary data. The presence of this outlier in the data deteriorates the performance of the detectors. In that case, $\hat{\Lambda}_{L R-F P E}$ achieves the best robustness. 

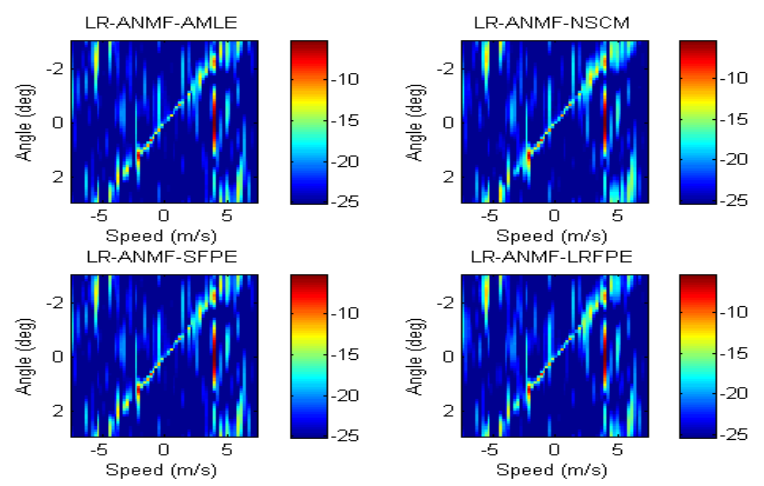

Fig. 3. Output detector of $\hat{\Lambda}_{A-M L E}$ (left up), $\hat{\Lambda}_{A-N S C M}$ (right up), $\hat{\Lambda}_{S-F P E}$ (left down), $\hat{\Lambda}_{L R-F P E}$ (right down). $K=100$.
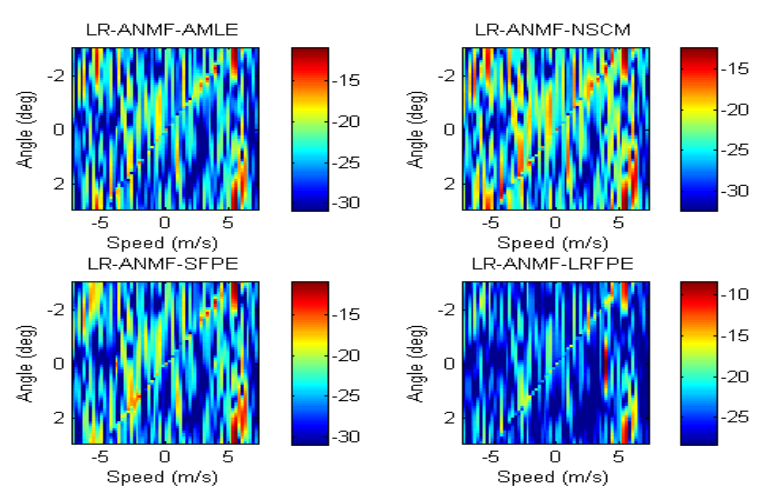

Fig. 4. Output detector of $\hat{\Lambda}_{A-M L E}$ (left up), $\hat{\Lambda}_{A-N S C M}$ (right up), $\hat{\Lambda}_{S-F P E}$ (left down), $\hat{\Lambda}_{L R-F P E}$ (right down). $K=250$ with outlier in secondary data.

\section{APPLICATION TO REAL DATA}

The performance of LR-ANMF detectors is tested on a real STAP data set. The STAP data are provided by the French agency DGA/MI: the clutter is real but the targets are synthetic. The number of sensors is $Q=4$ and the number of coherent pulses is $P=64$. The center frequency and the bandwidth are respectively equal to $f_{0}=10 \mathrm{GHz}$ and the bandwidth $B=5 \mathrm{MHz}$. The radar velocity is given by $V=100 \mathrm{~m} / \mathrm{s}$. The inter-element spacing is $d=0,3 \mathrm{~m}$ and the pulse repetition frequency is $f_{r}=1 \mathrm{kHz}$. The clutter rank, computed from Brennan Rule, is $R=45$ and the CNR is equal to $20 \mathrm{~dB}$. Targets with a Signal to Clutter Ratio (SCR) of $-5 \mathrm{~dB}$ is present.

Figure 3 presents the output of the different detectors for $K=100 \sim 2 R$. It illustrates the relatively equivalent performance of the detectors when there is no corruption. Figure 4 presents the output of the different detectors for $K=250$ with data containing the target as an outlier and shows the robustness of $\hat{\Lambda}_{L R-F P E}$.

\section{CONCLUSION}

In this paper, we have presented numerical performances of LR-ANMF build from different CSP estimators. The CFAR property of the adaptive LR detectors have been observed for every estimator. Results show that the best performances in terms of PD/PFA is achieved with $\hat{\Lambda}_{A-M L E}, \hat{\Lambda}_{S-F P E}$ and even $\hat{\Lambda}_{S C M}$. However robustness tests and a real data set have also illustrated the interest of the LR-FPE, which seems to propose a good trade off between performance and robustness for LR adaptive detection.

\section{REFERENCES}

[1] R.S. Raghavan, "Statistical interpretation of a data adaptive clutter subspace estimation algorithm," IEEE Trans. on Aero. and Elec. Syst., vol. 48, no. 2, pp. $1370-1384,2012$

[2] A. Breloy, L. Le Magoarou, G. Ginolhac, F. Pascal, and P. Forster, "Maximum likelihood estimation of clutter subspace in non homogeneous noise context," in Proceedings of EUSIPCO, Marrakech, Morocco, September 2013.

[3] A. Breloy, G. Ginolhac, F. Pascal, and P. Forster, "Robust estimation of the clutter subspace for a low rank heterogeneous noise under high clutter to noise ratio assumption," in Proceedings of ICASSP, Submitted, Florence, Italy, May 2014.

[4] I. Kirsteins and D. Tufts, Rapidly adaptive nulling of interference, chapter 6, High Resolution Methods in Underwater Acoustics. M. Bouvet and G. Bienvenu, Springer-Verlag, New York, 1991.

[5] I.S. Reed, J.D. Mallett, and L.E. Brennan, "Rapid convergence rate in adaptive arrays," IEEE Trans. on Aero. and Elec. Syst., vol. AES-10, no. 6, pp. 853 - 863, November 1974.

[6] G. Ginolhac, P. Forster, J.P. Ovarlez, and F. Pascal, "Spatio-temporal adaptive detector in non-homogeneous and low-rank clutter," in Proceedings of ICASSP, Taipei, Taïwan, april 2009.

[7] E. Ollila, D.E. Tyler, V. Koivunen, and H.V. Poor, "Complex elliptically symmetric distributions: Survey, new results and applications," Signal Processing, IEEE Transactions on, vol. 60, no. 11, pp. 55975625, 2012.

[8] F. Pascal, Y. Chitour, J.P. Ovarlez, P. Forster, and P. Larzabal, "Existence and characterization of the covariance matrix maximum likelihood estimate in spherically invariant random processes," IEEE Trans on Sig. Proc., vol. 56, no. 1, pp. 34 - 48, January 2008.

[9] A. Wiesel, "Unified framework to regularized covariance estimation in scaled Gaussian models," Signal Processing, IEEE Transactions on, vol. 60, no. 1, pp. 29-38, 2012.

[10] O. Besson and Y. Abramovich, "Regularized covariance matrix estimation in complex elliptically symmetric distributions using the expected likelihood approach - part 2: The under-sampled case," Signal Processing, IEEE Transactions on, vol. PP, no. 99, pp. 1-1, 2013.

[11] M. Rangaswamy, F.C. Lin, and K.R. Gerlach, "Robust adaptive signal processing methods for heterogeneous radar clutter scenarios," Signal Processing, vol. 84, pp. 1653 - 1665, 2004.

[12] J. Ward, "Space-time adaptive processing for airborne radar," Tech. Rep., Lincoln Lab., MIT, Lexington, Mass., USA, December 1994.

[13] L. E. Brennan and F.M. Staudaher, "Subclutter visibility demonstration," Tech. Rep., RL-TR-92-21, Adaptive Sensors Incorporated, March 1992.

[14] G. Ginolhac, P. Forster, F. Pascal, and J. Ovarlez, "Derivation of the bias of the normalized sample covariance matrix in a heterogeneous noise with application to low rank stap filter," Signal Processing, IEEE Transactions on, vol. 60, no. 1, pp. 514-518, Jan 2012. 